\title{
Il lessico delle cure e della farmacologia in papiri medici da Ossirinco
}

\author{
Daniela Fausti
}

Nell'ambito dei papiri provenienti da Ossirinco i testi medici occupano certamente un posto rilevante e il loro studio ci può permettere di tracciare un quadro dell'attività medica, della sua importanza in questa zona e della posizione del medico nella società ${ }^{1}$. Il fondamentale studio del 2007 di Peter Parsons, City of the Sharp-Nosed Fish, in particolare pp. 177-852, ci presenta una situazione in cui circolavano testi di tipo letterario di autori noti come Ippocrate, Dioscoride, Galeno, Oribasio e anche altri, che testimoniano la presenza della cultura medica canonica, cui si aggiungono una serie di adespoti di argomento medico, lettere e rendiconti ufficiali di medici pubblici (denominati $\delta$ nó iatpoí, cf. ad es. P.Oxy. XLIV 3195 del 331 d.C.; LIV 3729 del 307 d.C.; LXIV 4441 del 316 d.C.) $)^{3}$. Sono da segnalare, in quanto importanti per la figura del medico libero professionista e di un databile al IV d.C.) del medico Eudaimon a madre, nonne e sorella, che contiene la richiesta di strumenti professionali e medicamenti (perché è lontano da Ossirinco) e l'elenco dei prodotti della bottega ben fornita di un 'farmacista' situata proprio ad Ossirinco (XXXI 2567 del 18 maggio 253). Circolavano inoltre numerosi testi tecnici come ricettari, prescrizioni e manuali di farmacologia, dalla cui analisi possiamo ricavare un interessante lessico specifico: nome di rimedi usati e loro origine (per lo più vegetale), patologie a cui sono destinati, posologia, metodi di preparazione e loro descrizione, modi di somministrazione (impiastri, bevande, colliri, unguenti e oli profumati), cioè una panoramica sui metodi di cura non chirurgici.

Alcuni di questi documenti sono noti da tempo e sono stati oggetto di vari studi, citiamo soltanto il P.Oxy. VIII 1088 del I d.C., "Medical Receipts", edito per la prima volta da A. S. Hunt nel $1911^{4}$, ma dopo la pubblicazione nel 2014

\footnotetext{
${ }^{1}$ Un quadro generale in Andorlini Marcone, Apporto.

${ }^{2}$ Ora in traduzione italiana: Parsons, Ossirinco, 221-32.

${ }^{3}$ Sui medici pubblici cf. lo studio di Manfredi, Referti, e Reggiani, Report.

${ }^{4}$ Ripreso da Lundon, POxy 1088. Il testo si articola in 69 righe che contengono rimedi per curare ferite, flussi, ulcere, contusioni, febbri ecc.; l'esposizione è quella classica di questo tipo di scritti, dove spesso i rimedi elencati sono introdotti dall'espressione $\alpha{ }^{\prime} \lambda \lambda_{0}$ (cf. rr. 52 e 56), che
} 
del vol. LXXX degli Oxyrhynchus Papyri ${ }^{5}$ si è notevolmente ampliato il numero dei testimoni provenienti da un unico luogo (il che ci dà la possibilità di osservare l'attività medica in un'area geografica omogenea) e distribuiti in un periodo di tempo piuttosto lungo (dal I al VI d.C.). Per ciò che riguarda le patologie si può rilevare che sono presenti malattie degli occhi $(5240,5241)$, a cui sono dedicate ricette per colliri (5243); febbri dovute a malattie acute (5233, 5238); cancrena (5236), ulcere serpeggianti (5250); emorroidi (5232); ulcerazioni alle gengive per cui si dà la composizione di una polvere dentifricia (5244). Il nome del paziente è quasi sempre trascurato, tranne nei rapporti ufficiali, ad es. in caso di ferite (5254 l'interprete Nilo, 5255 un sovrintendente, 5256 Zoilo ferito, 5257 Serapione con una ferita alla testa) e le ricette sono per lo più anonime, a parte qualche caso $(5244,5246,5248,5249,5250)$ dove compare il nome dell'autore, rispettivamente Giuliano di Cesarea, Massimo il tagliapietre, Efestione, Amoitas, Eraclide.

Accanto a prescrizioni che si ispirano alla medicina razionale, come un ricettario miscellaneo (5247) per malanni di vario tipo (dalla tosse al morso dell'aspide) o ricette per infiammazioni suppurative e ulcere della testa (5248), troviamo stringate liste di ingredienti in gran parte di origine vegetale $(5252 \mathrm{e}$ 5253) o indicazioni pratiche per la preparazione di oli profumati (5242); inoltre sei brevi ricette di tipo medico-magico (5245) attestano la presenza di forme di medicina popolare. Ulteriori conferme sull'argomento sono venute dalla pubblicazione nel 2016 del volume LXXXII, con la sezione V, "Magic and Medicine" (nn. 5303-5315) 6 . Prendendo in considerazione anche un gruppo di testi pubblicati nel volume LXXIV del 2009, alcuni ricettari (4976, 4977, 4978, 4979) e un manuale di farmacologia (4975) databili dal II al III d.C. è possibile ampliare il quadro generale sulle conoscenze farmacologiche.

All'interno del gruppo di papiri pubblicati nel volume LXXX possiamo constatare anzitutto la presenza di testi identificabili con opere di autori ben noti come Ippocrate (si segnalano da 5219 a 5223: Aphorismi, De alimento, De liquidorum usu, De mulierum affectibus I, Epidemiae I, ${ }^{7}$ Prognosticum) e per Galeno tre esempi da 5227 a 5229: De locis affectis, De sanitate tuenda, Commentarium in Hippocratis Epidemiarum librum III. È forte l'interesse per la farmacologia, ben documentato nei testi adespoti e ulteriormente confermato dalla presenza di tre papiri che conservano il testo dioscorideo: 5224, identificabile con De materia medica I 61,2, sull'unguento di cinnamomo e I 6364,1, su quello di malabatro e la mirra, del IV d.C.; 5225 del II d.C. che riporta

\footnotetext{
indica una sequenza di ricette sullo stesso tema.

${ }^{5}$ Con prefazione di V. Nutton, pp. V-VI.

${ }^{6}$ Collocabili cronologicamente dal II al VI d.C., editi da F. Maltomini, tranne i P.Oxy. 5313 e 5314, editi da L. Tagliapietra, "Amuleti cristiani”.

${ }^{7}$ Fra l'altro il primo papiro pubblicato di Epidemie I.
} 
passi (III 17-18) dedicati ad acanto, acanto spinoso e ononide e infine 5226 del III d.C. su finocchio selvatico, dauco, piretro e rosmarino (III 71-74). Questi reperti inoltre raddoppiano il numero delle copie a noi finora note relative a Dioscoride e fanno del De materia medica il testo medico conosciuto meglio rappresentato su papiro ${ }^{8}$ insieme agli Aforismi di Ippocrate. Per quel che si può vedere il testo non era accompagnato da illustrazioni.

Alla tematica farmacologica appartiene anche il 5230 del II d.C., da ricondurre almeno in una sua parte all'opera Narthex di Heras di Cappadocia, nome illustre della farmacologia antica ${ }^{9}$. Il papiro conserva (fr. 1) la ricetta di un impiastro per ulcere maligne e loro cicatrizzazione a base di cera, resina abbrustolita, raschiatura di ruggine, polvere d'incenso, olio d'oliva, aceto. Le indicazioni di quantità dei singoli componenti sono precise, libbre, once, kotylai e staio. Questa ricetta è contenuta nel De compositione medicamentorum per genera V 2 (XIII 765-766 K.) di Galeno che la attribuisce ad Heras, peraltro il resto della sequenza non corrisponde, il che fa pensare che l'autore del papiro possa aver copiato dal manuale di Heras o da una compilazione, come quella di Galeno, che ne riportava estratti.

\section{Rimedi e strumenti a disposizione del medico, senza dimenticare i libri}

Quali siano i tre elementi più importanti a disposizione del medico per esercitare bene la sua professione si evince dai passi introduttivi del De indolentia, dove Galeno, raccontando dell'incendio di Roma che nel 192 d.C. distrusse le sue $\dot{\alpha} \pi \circ \theta \hat{\eta} \kappa \alpha \iota$ della Via Sacra, ricorda con abbondanza di particolari la perdita di una

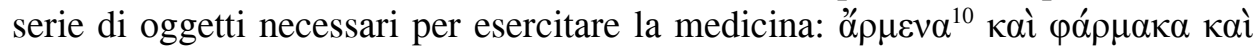
$\beta \imath \beta \lambda i ́ \alpha$, di cui possiamo individuare la presenza anche nei papiri di Ossirinco. Un punto particolarmente interessante è quello dove si rammarica di aver perso farmaci di ogni genere e strumenti che aveva lui stesso inventato "i cui modelli plasmati in cera consegnavo ai fabbri" e che non si potevano riavere se non con molto tempo e fatica; questo valeva anche per i libri, sia quelli composti personalmente sia quelli copiati ed emendati di sua mano (parr. 4-6 = p. 3,3-16 Boudon-Millot/Jouanna).

Per constatare l'importanza di questi oggetti nella città "del pesce dal naso aguzzo", possiamo riprendere due esempi già citati, quello del medico Eudaimon e quello del 'farmacista' del P.Oxy. 2567. Il medico nella lettera contenuta in P.Oxy. LIX 4001 del IV d.C. edito nel 1992 da H. G. Ioannidou ${ }^{11}$, dopo le usuali formule di saluto a madre, nonne e sorella, invocazione alla divina provvidenza

\footnotetext{
${ }^{8}$ Cf. il commento nell'edizione di Leith, p. 15.

${ }^{9}$ Ipotesi avanzata dall'editore Leith, p. 38.

${ }^{10}$ Sul termine $\alpha$ $\rho \mu \varepsilon v \alpha$ per "strumenti", cf. Andorlini, Strumenti, 239 ss.

${ }^{11}$ Per testo, traduzione italiana e osservazioni varie, cf. il lavoro di Bonati, Lessico, 304-5.
} 
(siamo in epoca cristiana) e notizie di argomento familiare, dal r. 20 delinea un quadro significativo dal punto di vista professionale.

Eudaimon stava probabilmente lavorando fuori sede in un villaggio della $\chi \omega ́ \rho \alpha$ e scrive al suo i $\alpha \tau \rho \varepsilon \hat{o} v$ ad Ossirinco chiedendo che gli mandino una serie di strumenti, segnalando nel contempo degli errori nella spedizione precedente. Infatti mentre da casa avevano dichiarato l'invio di cinque libri, lui ha avuto da Elena la ricamatrice una sacca contenente soltanto quattro libri ( $\mu$ óv $\alpha \delta^{-} \beta 1 \beta \lambda i^{\prime} \alpha$ ),

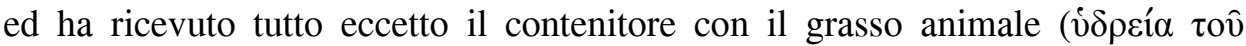

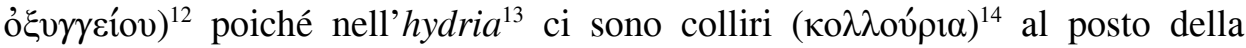
sugna. Questa è la sola testimonianza papiracea finora conosciuta dell'uso dell'v́ $\delta \rho i ́ \alpha$ come contenitore farmaceutico; la motivazione di questa scelta si può capire ricordando che il microcontenitore doveva essere ad imboccatura stretta e manico verticale, adatto ad essere accuratamente chiuso e poi spedito ${ }^{15}$. Nelle ultime righe lo scrivente chiede che gli sia inviato anche l'astuccio di bronzo ( $\tau$ ò

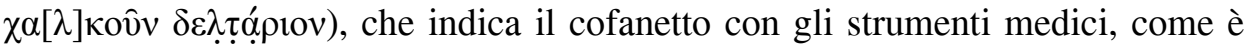
stato dimostrato già da Fischer nel $1997^{16}$, poi il contenitore per l'acqua calda

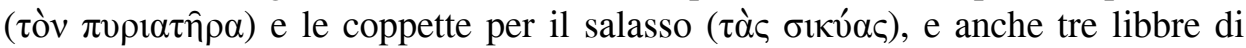
colliri "misti" e astringenti.

Il motivo della richiesta dell'astuccio può trovare spiegazione nella frase: "affinché io possa (far ?) fabbricare ( $\pi$ oiń $\sigma \omega)$ altri strumenti", dando al verbo un valore causativo ${ }^{17}$ per cui la manifattura della strumentazione sarebbe affidata a un fabbro o ad un'officina di artigiani, che dovevano copiare dai modelli di

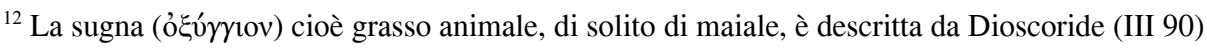
come sostanza da mescolare con elementi vegetali per poterli spalmare. L'impiego farmaceutico dell'ỏ $v^{\prime} \gamma \gamma$ iov, è ampiamente confermato dai trattati di medicina, mentre nei papiri sembra avere una sola occorrenza sicura oltre a questa, all'interno di una delle quattro ricette per unguenti trasmesse in SB XXIV 15917,21 (II d.C., Ankyron [M-P ${ }^{3}$ 2398.12; LDAB 4702]). Cf. Bonati, Lessico, 168. Normalmente i colliri si presentavano in forma di panetti solidi, per poter essere

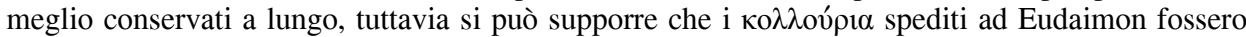
stati anticipatamente diluiti con opportune sostanze probabilmente per agevolare il lavoro del medico, mandando un prodotto semilavorato.

${ }^{13}$ Cf. Bonati, Lessico, 157-9, per un'analisi dei vari significati di vo $\delta \operatorname{ci}_{\alpha}$, brocca di vari tipi; si evidenziano alcune testimonianze che documentano l'uso di (micro)contenitori definiti vo $\delta \rho^{\prime} \alpha /$

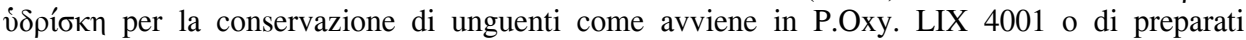
medicinali di consistenza semiliquida (pp. 168 e 175).

${ }^{14}$ Pomate/unguenti per gli occhi, che dimostrano l'interesse per la cura delle malattie oftalmiche.

${ }^{15} \mathrm{Cf}$. Bonati, Lessico, 168.

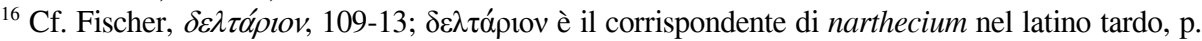

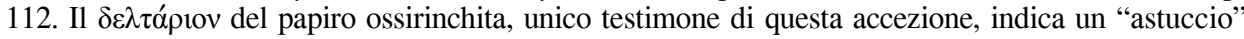
rettangolare a cerniera con due ante, il cui nome è determinato proprio dalla morfologia dell'oggetto, che rimanda alla forma del codex aperto. Si tratta quindi di un termine tecnico del lessico dei contenitori di uso medico, che ribadisce, inoltre, la familiarità degli specialisti coi materiali scrittori, visto che il libro tecnico costituiva un elemento essenziale per l'apprendimento e lo svolgimento della professione. Cf. Bonati, Lessico, 190.

${ }^{17}$ Bonati, Lessico, 311.
} 
Eudaimon $^{18}$. Questa interpretazione è la più sostenibile, in quanto è probabile che il contributo dei medici alla creazione degli ó $\rho \mu \varepsilon v \alpha$ riguardasse più che la fabbricazione materiale, l'invenzione di nuovi strumenti o la modifica di quelli preesistenti, come si desume dal passo sopracitato di Galeno. Oltre al termine $\delta \varepsilon \lambda \tau$ óplov presente nel papiro possiamo segnalarne un altro ugualmente raro: $\pi v \rho ı \tau \tau$ ́ $\rho$ "borsa dell'acqua calda", unico caso presente nei papiri medici e testimoniato in letteratura soltanto da Sorano (III 10,3) ${ }^{19}$. La consuetudine di richiedere per lettera in caso di lontananza da casa i mezzi necessari ad esercitare la professione e la borsa che li contiene è testimoniata non solo dal papiro ossirinchita, ma anche dal P.Strasb.Copte inv. $563^{20}$, datato fra il VI/VII sec., purtroppo di provenienza sconosciuta ${ }^{21}$. In questo caso lo schema della richiesta è molto semplice, il medico chiede al suo ignoto corrispondente di mandargli la sua benda, ${ }^{22}$ la tazza (cyathus), il sigillo rotondo, la sua borsa da medico $(\pi \eta ́ \rho \alpha)$, il bisturi e il cucchiaio di legno ${ }^{23}$. Il contenitore degli strumenti, la $\pi \hat{n} \rho \alpha$, doveva probabilmente essere di cuoio ed è finora questa l'unica attestazione con tale significato ${ }^{24}$. Qui non sono nominati i libri; invece, su un altro medico fuori sede che si preoccupa anzitutto per i suoi libri di uso professionale, ci testimonia il P.Ross.Georg. III 1 del III d.C.; lo scrivente si chiama Marco e si rivolge alla madre pregandola di scuotere la polvere da "i libri di medicina", $\tau \grave{\alpha}$ i $\alpha \tau \rho \iota \alpha \grave{~}$ $\beta v \beta \lambda i ́ \alpha$, e di toglierli dalla nicchia vicino alla finestra dove li aveva lasciati prima di partire (rr. 17-19). Come spesso accade i papiri documentari ci riportano un vivace quadro della vita di tutti i giorni, ma talvolta ci presentano casi di difficile interpretazione come ad es. P.Oxy. 4979 del II/III d.C. ${ }^{25}$ che conserva una breve lista, dove i primi due termini potrebbero rimandare ad oggetti usati nell'attività chirurgica e gli altri tre alla terapia farmacologica.

Esaminiamo il brevissimo testo:

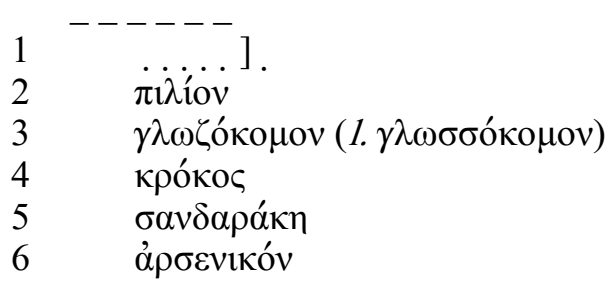

\footnotetext{
${ }^{18}$ Cf., sul tema, Ghiretti, Luoghi, 104-5.

${ }^{19} \mathrm{Su}$ questo oggetto cf. Andorlini, Borse.

${ }^{20}$ Ovvero GMP II 10.

${ }^{21} \mathrm{Cf}$. le osservazioni di Fischer Bovet, Letter.

${ }^{22}$ Cioè il materiale da cui ricavare le bende.

${ }^{23}$ Serviva a distribuire, misurare la quantità dei medicinali. Un oggetto di uso pratico che diventa unità di misura, cf. Reggiani, Liquidi, 114.

${ }^{24}$ Cf. Fischer Bovet, Letter, 163 e Bonati, Lessico, 314.

${ }^{25}$ Cf. l'ed. di D. Leith del 2009.
} 
Gli ultimi tre elementi, croco, realgar e arsenico ${ }^{26}$ hanno una chiara caratterizzazione: sono usati in medicina o per le tinture ${ }^{27}$. Il croco (zafferano) ha una tradizione medica che risale agli scritti ippocratici: è usato ad es. per malattie degli occhi in Epidemie II 5,22 ed è presente nelle ricette per colliri di P.Oxy. VIII 1088,6 e P.Strasb.Gr. inv. 90,4 (manuale di oftalmologia); Dioscoride gli attribuisce numerose proprietà: digestive, emollienti, astringenti, diuretiche (I 26); era perciò molto richiesto come testimonia anche un ostrakon che conserva il messaggio di un medico al fratello con richiesta di spedizione di croco ad uso terapeutico (O.Claud. II 220, datazione fra il 137 e il 145) ${ }^{28}$. Ai due solfuri si riconoscono proprietà caustiche e purificanti (Dioscoride V 104-105); l'arsenico è prescritto in P.Oxy. 1088,28 per la cura dei polipi nasali, il realgar è presente nei trattati ginecologi del Corpus (ad es. in De mulierum affectibus, II $200=$ VIII 382 L.) e insieme al croco in P.Ryl. III 531v,47-48 è impiegato per cure stomatologiche. Tutti gli elementi sono più volte presenti in Galeno. Bisogna dunque esaminare gli altri due termini della lista per cercare di verificare se si tratta davvero di un contesto medico.

Il $\pi i \lambda$ íov (notare l'uso del diminutivo) è un cappellino, cioè un oggetto di vestiario, ma fra $\mathrm{i}$ vari possibili confronti già segnalati ${ }^{29}$ due esempi in particolare vanno in direzione dell'uso medico; il termine in Sorano De fasciis 2 indica un particolare tipo di bendaggio della testa e in maniera più stringente possiamo considerare Platone Respublica 406d dove si parla di $\pi 1 \lambda i \delta 1 \alpha$ (ugualmente un diminutivo) che i medici mettevano sulla testa dei pazienti per poter applicare i medicamenti. In contesto chirurgico $\gamma \lambda \omega \sigma \sigma o ́ \kappa o \mu o v$ indica una specie di gabbia per proteggere le gambe fratturate; Galeno (in Hipp. Fract. II 64 = XVIII.2 501-505 K.) la descrive come una scatola (aperta in cima e in fondo) che bloccando la gamba infortunata impediva movimenti che la danneggiassero. All'interno era imbottita con lana e bende; poteva quindi figurare fra gli strumenti tenuti a disposizione del medico. Questi primi due sostantivi però potrebbero avere significati più banali ed essere rispettivamente un capo di vestiario e una cassetta per oggetti d'uso. Tuttavia l'inserimento in un contesto medico renderebbe l'elenco più coerente ${ }^{30}$. Si può a proposito ricordare che già nel Corpus si consigliava ai dottori di tenere a disposizione una scorta di medicinali, farmaci preparati nel modo dovuto, secondo i relativi generi, freschi o trattati in maniera da conservarsi (De decenti habitu $10=$ IX 238 L.).

\footnotetext{
${ }^{26}$ I solfuri di arsenico erano noti dall'antichità e sono distinti dal colore, rosso il primo, giallo il secondo.

${ }^{27}$ Cf. vari esempi riportati da Leith, p. 88.

${ }^{28}$ Il medico Psenpaapis chiede al fratello di procurarsi lo zafferano (croco) dal medico e inviarglielo. Cf. Bonati, Lessico, 302.

${ }^{29}$ Cf. l'ed. di Leith, p. 87.

${ }^{30}$ Sulla compresenza negli iatreia di strumenti e sostanze medicamentose cf. Ghiretti, Ambulatorio, 87.
} 


\section{I libri: trattati generali e ricettari}

Eudaimon ed altri medici nelle lettere nominano i libri e attraverso i reperti papiracei possiamo ipotizzare il tipo di materiale scientifico a disposizione. Anzitutto trattati di medicina di autori fondamentali quali Ippocrate, Dioscoride, Galeno (vd. supra), ma nel gruppo dei testi adespoti possiamo segnalare manuali redatti in forma di questionario come il $5238^{31}$ del II/III d.C. a contenuto terapeutico e in parte prognostico, con notevole attenzione alle febbri e sintomi associati. Rimedi consigliati: acqua calda e fredda, olio di oliva, bende di lana e di lino, spugne, embrocazioni di olio di oliva ${ }^{32}$, clistere e flebotomia; un sistema espositivo simile con definizioni mediche in forma catechistica su apostema, emorragia, enterocele, idrocele è utilizzato dal papiro 5239 (II/III d.C.).

Due testi riguardano le malattie degli occhi: il primo (P.Oxy. 5240, del I d.C.), descrive interventi chirurgici per affezioni oftalmiche; poteva trattarsi di un manuale per uno studente o per un pubblico colto. Dal punto di vista linguistico è interessante l'uso di diminutivi per i nomi degli strumenti, che ci testimoniano il contesto quotidiano colloquiale ${ }^{33}$. Abbiamo infatti: "piccolo uncino"

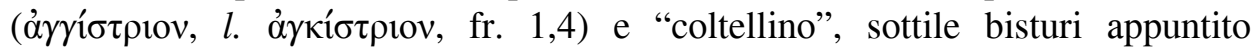
( $\sigma \mu \imath \lambda \alpha$ ópıv, fr. 1,5) e probabilmente un neologismo nel fr. 3,6, $\pi \varepsilon \rho \imath \ddot{\alpha} \alpha ́ \delta ı v$ strumento "per tagliare intorno" (interpretazione suggerita da I. Andorlini ${ }^{34}$ ), che

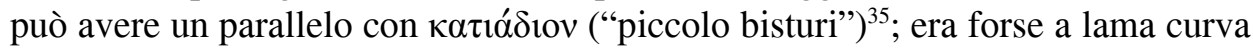
ed usato per la chirurgia oculare.

L'altro papiro sulle affezioni oculari è il 5241: il fr. 1 tratta della cataratta

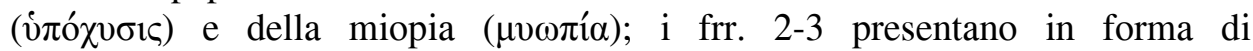
questionario definizioni di affezioni oculari. Questo fa pensare che dovesse far parte di un manuale per medici o studenti di medicina. Si conoscono circa 20 papiri medici redatti in questa forma (che favoriva l'apprendimento mnemonico) e databili dal II al IV d.C.; tre riguardano l'oftalmologia ${ }^{36}$. Di carattere più generale è il 5237 del II d.C., manuale a contenuto fondamentalmente terapeutico; espone la teoria che le malattie sono dovute al caldo e al freddo e questi stessi elementi forniscono i mezzi per contrastarle. L'attenzione alle febbri è legata al fatto che sono una manifestazione di malattie causate da caldo o freddo; dal punto di vista dei rimedi l'unica terapia di cui rimane traccia è la

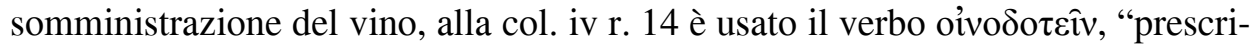

\footnotetext{
${ }^{31}$ I testi di seguito esaminati, 5238 e 5239, sono stati editi da D. Leith.

${ }^{32}$ Preparato medicinale liquido, a base oleosa o grassa, destinato a essere applicato su una parte dolente del corpo.

${ }^{33}$ Cf. le osservazioni di Reggiani, Liquidi, 114.

${ }^{34}$ Riportata dall'editrice Hirt, p. 103.

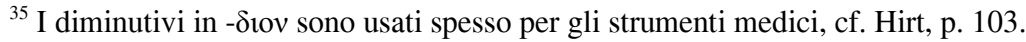

${ }^{36}$ Cf. Hirt, pp. 105-6 e relativa bibliografia.
} 
vere vino" (cf. Herod. Med. in Orib. V 27,15). Il notevole interesse per la cura delle febbri è usuale, quello per malattie degli occhi ${ }^{37}$ doveva essere causato dal fatto che queste ultime erano frequenti per il clima caldo e l'ambiente circostante caratterizzato dalla sabbia.

\subsection{Farmacologia}

Anche per la farmacologia c'erano a disposizione manuali di cui possiamo considerare un esempio, un testo anonimo del II d.C., il P.Oxy. $4975^{38}$. Le ricette sono state approssimativamente ordinate seguendo la successione delle parti malate che si intendeva trattare; si inizia con la zona dell'inguine, dei genitali poi seguono rimedi per difficoltà respiratorie rovesciando il più tradizionale ordine a capite ad calcem. Somiglianze di presentazione grafica si possono notare con P.Oxy. 1088 (vd. supra) poiché abbiamo una serie di ricette separate dalla paragraphos seguita da un titolo in ekthesis.

I rimedi proposti sono in maggioranza di derivazione vegetale: uva passa dolce, ruta e foglie di alloro con miele, cipresso verde mescolato con vino egiziano, semi di nasturzio, giusquiamo, pepe bianco e issopo, ricino, abrotano, ma anche cenere ricavata da pezzi di lino bruciato come antinfiammatorio per i genitali, e al r. $7 \sigma \pi$ ó $\delta$ เov (ossido - forse di zinco) e nardo per l'interno delle cosce; il miele viene usato come eccipiente. I medicamenti sono tutti molto diffusi, si nota però la prescrizione di vino egiziano, che era molto pregiato e tradizionalmente destinato ad una consumazione elitaria. Tracce antichissime se ne sono trovate in anfore nella tomba di Tutankhamon ${ }^{39}$.

I P.Oxy. 4977 e 4978, entrambi databili fra il II e III d.C., presentano la struttura semplice dei ricettari. Nel primo la prescrizione meglio conservata riporta le indicazioni per un collirio e conferma l'interesse per le malattie degli occhi e anche la ricerca di un certo benessere del paziente: un collirio (quello fatto con le

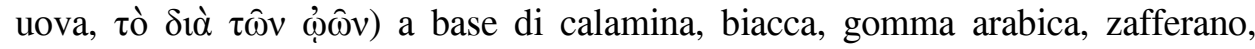
oppio $^{40}, 4$ uova che rendono il collirio "delicato" ( $\left.\tau \rho v \varphi \varepsilon \rho o ́ v\right)$; le quantità sono date in dracme. Questi colliri 'delicati' erano destinati a pazienti che soffrivano di affezioni oculari dolorose e croniche. Il P.Oxy. 4978 conserva tre ricette complete

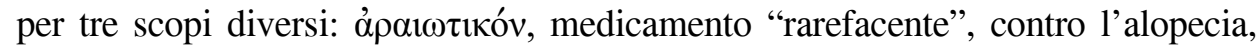
come si evince dal fatto che vengono usati semi di papavero ed escrementi di topo,

${ }^{37}$ Queste potevano infatti aver effetti invalidanti; un esempio molto noto è P.Oxy. I 39 edito da Grenfell e Hunt nel 1898; nel 52 d.C. Trifone il tessitore è esentato dal servizio militare o da una tassa, perché ci vede poco in quanto affetto da cataratta (duplicato in II 317): $\dot{\pi}\langle\langle\kappa \varepsilon\rangle \chi v \mu \varepsilon ́ v o \varsigma$ ỏ $\lambda i ́$ íov $\beta \lambda \varepsilon ́ \pi \omega v$. Per una storia di questo personaggio attraverso i papiri, cf. Parsons, Ossirinco, 263-7.

${ }^{38}$ Edito nel vol. LXXIV del 2009 da D. Leith, come pure i due successivi.

${ }^{39}$ Cf. Guash-Jané, Wine, 851-8.

${ }^{40}$ Con effetto anestetico e analgesico. 
tipico rimedio contro la caduta dei capelli ${ }^{41}$; in aggiunta, un olio profumato doveva servire sia come eccipiente per il medicamento sia per eliminare il cattivo odore

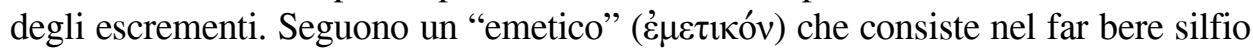
in acqua bollita; secondo Galeno (De antidotis XIV $143 \mathrm{~K}$.) bere silfio ${ }^{42}$ con un liquido funzionava come emetico per quelli che avevano bevuto sangue di toro

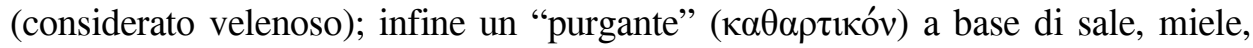
aceto e acqua.

\subsection{Ricette 'firmate'}

Qualche ricetta ci ha conservato il nome dell'autore e possiamo individuare nel volume LXXX cinque esempi, di cui tre ${ }^{43}$ ci consentono di fare qualche interessante riflessione di tipo storico-sociale. Il P.Oxy. 5246 del II/III d.C., contiene due ricette, la prima contro la podagra, la seconda di argomento non chiaro. L'aspetto interessante della prima è che lo scrivente dichiara di averla avuta da Massimo il tagliapietre ( $\left.\lambda \alpha \xi_{\text {ovo }}\right)$, quindi non un professionista, ma un portatore di conoscenze tradizionali. Questa specificazione ricorda l'abitudine di Teofrasto di avere fra i suoi informatori anche artigiani vari ${ }^{44}$. La prescrizione è a base di semi di lino e olio, ma è da notare che il redattore contrappone a ciò

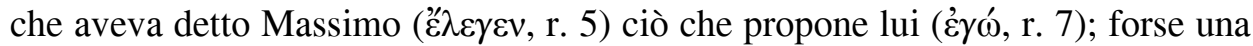
critica implicita da parte di un esperto. Il papiro 5249 del III d.C. riporta due prescrizioni, la prima, mutila, riguarda affezioni della pelle (cf. P.Oxy. 1088,1418), come si vede dall'uso di cantaridi e pece umida, la seconda è definita come

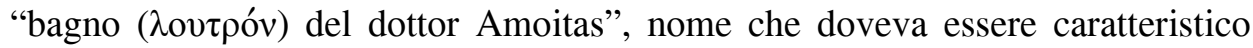
della zona di Ossirinco (cf. LVIII 3929 del 250 d.C.). Anche qui le malattie degli occhi sono al centro dell'attenzione: i malati si dovevano lavare gli occhi con questo farmaco a base di calamina ${ }^{45}$, rame bruciato, oppio, erica, mirra, acacia, gomma arabica; la quantità è indicata in dracme. I 7 ingredienti si ritrovano nella preparazione di colliri áxápı $\sigma \tau \alpha$ ("aspri, sgradevoli”), in Galeno, Comp.med.loc. 4,7 (XII 749,13-750,11 K.), che si contrapponevano a quelli “delicati” (

\footnotetext{
${ }^{41}$ Cf. sulla definizione di alopecia Ps.Gal. Def.med. 314 (XIX 431 K.); sul rimedio, cf. Dsc. II 80,5; Plin. N.H. XXIX 106.

${ }^{42}$ Cf. sul silfio Dsc. III 80; sulla storia e gli usi della pianta cf. Roselli, Silfio, 11-20 e Braccini, Silfio, 161-75.

${ }^{43}$ Questi tre sono stati editi da M. Hirt. Ricordiamo rapidamente gli altri due: in 5248 del II/III d.C. (ed. Hirt) è citato Efestione, nome abbastanza comune, ma non si conoscono medici di fama così chiamati; in 5250 del III d.C. (ed. W. B. Henry) si parla del rimedio di Eraclide per le ulcere

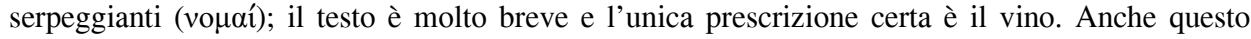
nome è abbastanza comune, difficile però pensare ad Eraclide di Taranto.

${ }^{44}$ Teofrasto ad es. ricorda le testimonianze dei boscaioli (H.P. III 3,7; IV 13,1) e carbonai (H.P. IX 3,1$)$ cioè persone che hanno una conoscenza pratica derivata dall'esperienza.

${ }^{45}$ Silicato basico di zinco ( $\left.\alpha \alpha \delta \mu \varepsilon i ́ \alpha\right)$.
} 
consigliati ad es. in P.Oxy. 4977 (vd. supra).

Una ricetta di polvere dentifricia a scopo esclusivamente curativo per le gengive è contenuta nel 5244 del III d.C.; le gengive sono già "erose"

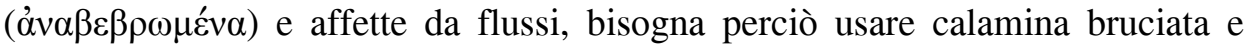

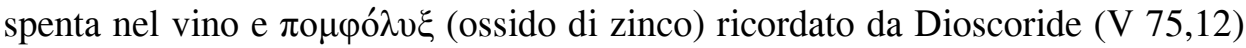
e più volte da Galeno (Simpl.temp.fac. XI 634 e 780; Comp.med.loc. XII 234 e 699,12-700,9 K., dove viene segnalato fra i farmaci non "pungenti") per le sue qualità astringenti, rinfrescanti, riempitive, purganti, adesive e in parti disseccanti, il che lo rendeva adatto per colliri ed impiastri per curare suppurazioni e ulcere ${ }^{46}$. Il rimedio porta il nome di Giuliano di Cesarea, forse il medico metodico attivo ad Alessandria nel II d.C. ${ }^{47}$

Il contenuto di un altro papiro, P.Oxy. 4976 del II/III d.C., fa pensare che ci potessero essere scambi di ricette fra dottori; anche se manca l'indicazione della malattia cui è destinata ${ }^{48}$, la ricetta ci fornisce la composizione di un prodotto fortemente aspro a base di felce maschio, scammonea, melanteria ${ }^{49}$, semi di nasturzio e nitro greco (E $E \lambda \lambda \varepsilon v i \kappa o ́ v)$; questo aggettivo riferito al carbonato di sodio si trova soltanto in due papiri (P.Genova II 62,11 e P.CtYBR inv. 1443 fr. C 4 e 6) e inoltre sappiamo che in Egitto c'erano ben tre luoghi specifici di provenienza del nitro ${ }^{50}$, ma ricordare che questo veniva dalla Grecia può voler sottolineare la ricerca di una qualità particolare, anche se già nei testi del Corpus Hippocraticum proprio i prodotti egiziani sono molto apprezzati ${ }^{51}$ e il nitron egizio è impiegato ad es. in Int. 26,31,48; d'altro canto, un passo di Plinio (N.H. XXXI 107) ricorda che in Macedonia ce ne era uno di qualità migliore.

Da segnalare per i primi due componenti la possibilità di scelta fra le quantità da somministrare: 8 o 4 dracme, $1 / 2$ dracma o una dracma e $1 / 2$; questa rara indicazione fa pensare che il papiro sia stato mandato da un dottore ad un altro o da un farmacista all'altro e che il ricevente abbia adattato le quantità (cf. P.Mert. I 12 , che testimonia uno scambio di ricette fra dottori) ${ }^{52}$. Del resto questa possibilità non contrasta con l'idea che la medicina procede per approssimazione. Nell'opera di Galeno, per citare un esempio illustre, c'è da un lato attenzione alla posologia nelle ricette, ma dall'altro è forte la convinzione dell'importanza

${ }^{46} \mathrm{Ne}$ esistevano due tipi, uno grigio/azzurro e grasso l'altro bianco e leggero; quest'ultimo era ricavato dalla mescolanza fra calamina e rame; così Dioscoride, V 75,1-7, e Plinio, N.H. XXXIV 128 ss., che lo inserisce fra i medicinali per gli occhi e per impiastri leggermente refrigeranti ed essiccanti.

${ }^{47}$ Parsons, Ossirinco, 225 ricorda la presenza di medici di questa scuola ad Ossirinco.

${ }^{48}$ Stando alla composizione il medicamento poteva essere un vermifugo (cf. Paul.Aeg. IV 57,13 e Dsc. Eup. II 68,2).

${ }^{49}$ Tintura nera usata dai calzolai e impiegata anche come farmaco, cf. Dsc. V 101.

${ }^{50}$ Cf. Leith, p. 80.

${ }^{51}$ Per ciò che riguardava il nitro, l'allume e il sale tradizionalmente i Greci consideravano migliori i prodotti egiziani, cf. Totelin, Recipes, 155.

${ }^{52}$ Cf. Leith, p. 79. 
del metodo stocastico, che rende dubbia la possibilità di quantificare i medicamenti nelle prescrizioni ${ }^{53}$.

\section{Il commercio dei prodotti medicinali}

La distribuzione e il commercio dei vari prodotti medicinali normalmente era affidata al $\varphi \alpha \rho \mu \alpha \kappa о \pi \omega ́ \lambda \eta \varsigma$, della cui attività abbiamo un esempio in P.Oxy. XXXI $2567^{54}$, che ci conserva la registrazione di uno stock di farmaci datata 18 maggio del 253 d.C.; la testimonianza è importante in quanto è l'unico caso di $\varphi \alpha \rho \mu \alpha \kappa o \pi \omega ́ \lambda \eta \varsigma$ documentato in papiri pubblicati ${ }^{55}$. Bisogna però qui ricordare un'attestazione al di fuori dei papiri documentari con l'esempio di P.Herc. 1674, la Retorica di Filodemo, col. xii, p. 67 (ed. Longo Auricchio), dove i venditori di farmaci vengono criticati attraverso un accostamento agli indovini per $\mathrm{i}$ falsi ragionamenti simili a quelli dei sofisti. Spesso questi personaggi erano infatti oggetto di giudizi negativi ${ }^{56}$. Diversa la situazione nel papiro ossirinchita, dove chi scrive è Aurelio Neottolemo, figlio di Dioscoro, $\varphi \alpha \rho \mu \alpha \kappa о \kappa \omega ́ \lambda \eta \varsigma$ della città di Ossirinco (r. 6) che si rivolge a tre concessionari del monopolio dell'allume nel Fayûm ${ }^{57}$, dichiarando in prima persona che su ordine di un altro ufficiale (l'amministratore di Hermes) farà un puntuale

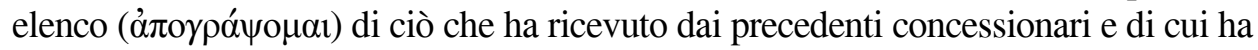
tutte le ricevute necessarie; si tratta delle regole di una consuetudine commerciale. Seguono la data e l'attestato di ricezione di un duplicato da parte di un funzionario, scritto da una seconda mano.

A partire dal r. 13 sono elencate queste sostanze e l'indicazione di quantità:

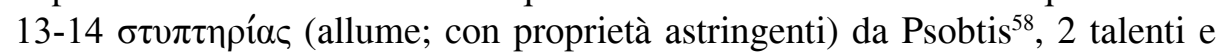
$1 / 2\left(\tau \alpha \dot{\lambda} \alpha \nu \tau \alpha \beta^{L^{-}}\right)$

$15 \sigma \chi \imath \sigma \tau \eta_{\varsigma}$ (allume fissile) 30 mine ( $\mu \nu \hat{\alpha} \varsigma \lambda^{-}$); secondo Plinio (N.H. XXXV 186) si tratta di un nome greco per un certo tipo di allume: schiston appellant Graeci.

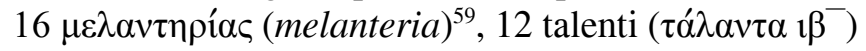

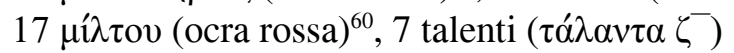

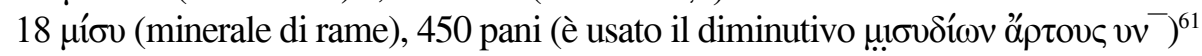

\footnotetext{
${ }^{53}$ Cf. Grimaudo, Misurare, 77-80.

${ }^{54}$ Edito da Parsons (1966).

${ }^{55}$ Secondo Totelin, Pharmacopōlai, 77.

${ }^{56} \mathrm{Il}$ passo mi è stato cortesemente segnalato da G. Del Mastro. Per un quadro generale sulla figura del $\varphi \alpha \rho \mu \alpha \kappa о \pi \omega ́ \lambda \eta \varsigma$, cf. Fausti, Farmacologia, 22-4 e relativa bibliografia.

${ }^{57} \mathrm{Si}$ tratta di ufficiali, stanziati ad Alessandria, che garantiscono che siano pagate le tasse sull'allume e altri minerali monopolio di stato. Cf. sull'argomento anche le testimonianze di P.Oxy. XVII 2116 del 229 d.C. e XII 1429 del 300 d.C.; sull'importante testimonianza del papiro ossirinchita sulla questione del monopolio dell'allume, cf. Kruse, Alaunmonopols, 523-47.

${ }^{58}$ La capitale della Piccola Oasi, da cui veniva trasportata a dorso di cammello nel Fayûm.

${ }^{59}$ Vd. n. 48; ha le stesse proprietà del $\mu$ í́vo, menzionato al r. 18.

${ }^{60}$ Dioscoride (V 96) ricorda quella di Sinope, con proprietà astringenti.
} 


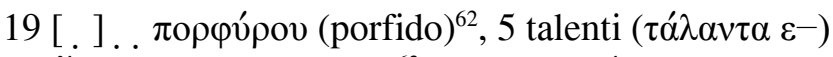

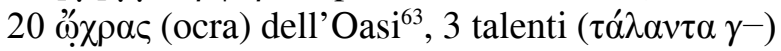

$21[\dot{\alpha}] \lambda \nu \kappa n ́ \varsigma$ (sale), 5 talenti ( $\left.\tau \dot{\alpha} \lambda \alpha v \tau \alpha \varepsilon^{-}\right)$

$22[$. . ] ]

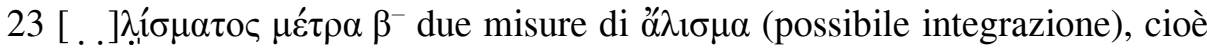
piantaggine $^{64}$. Era un antidoto contro la lepre di mare ${ }^{65}$, il rospo, l'oppio; serviva anche contro le coliche, la dissenteria, gli spasmi, somministrato alla quantità di due dracme. Qui vengono registrate due "misure"; il metron, era una misura per solidi (cf. Hdt. I 192,3) ${ }^{66}$ e, ammesso che si debba applicare alla pianta, vuol dire che veniva venduta a volume, cioè secondo il contenitore che veniva riempito. Gli elementi elencati sono usati in farmacologia (vd. note relative) e per attività industriali come la tintura; si può osservare che la quantità dei prodotti immagazzinati è generalmente notevole dato che il talento aveva un peso oscillante fra i 26 e $30 \mathrm{~kg}^{67}$.

Un confronto sulla vendita di farmaci, anche se non con una registrazione formale è testimoniato anche in P.Lond. II 356 del I d.C.: Procleios da Alessandria chiede in una lettera a Pecusis (che non è detto esplicitamente, ma si desume sia un $\varphi \alpha \rho \mu \alpha \kappa о \pi \hat{\lambda} \lambda \varsigma)$, di vendergli buoni $\varphi \alpha ́ \rho \mu \alpha \kappa \alpha$ per il suo amico Sotas, che glieli porterà ad Alessandria ${ }^{68}$.

Non sempre però la situazione commerciale è chiaramente riconoscibile, ci sono infatti reperti papiracei che ci hanno restituito nude liste di elementi che, in base all'osservazione che molti di solito venivano utilizzati per medicamenti, si ritiene siano una registrazione in vista di future vendite.

Il P.Oxy. 5252 del V d.C. ${ }^{69}$ incolonna, non in ordine alfabetico, il nome di 25 prodotti e la loro quantità; la maggior parte delle sostanze sono vegetali e

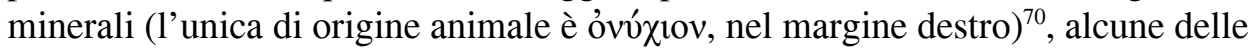

${ }^{61}$ Questo diminutivo si trova ad es. in ps. Galeno, De succedaneis XIX 736 K., dove vengono

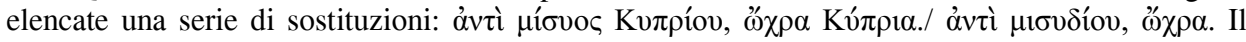
misy migliore era prodotto a Cipro: Dsc. V 100; era utilizzato per ulcere agli occhi.

${ }^{62}$ C'è una lacuna iniziale. Il porfido era una produzione tipica dell'Egitto, cf. Plin. N.H. XXXVI 57; da questo minerale si può estrarre il rame.

${ }^{63}$ L'ocra gialla secondo Dsc. V 93 aveva proprietà astringenti e disinfettanti.

${ }^{64}$ Pianta erbacea, cf. Dsc. III 152.

${ }^{65}$ Un mollusco diffuso in area mediterranea, che per difendersi lancia inchiostro; si credeva fosse tossico e addirittura mortale.

${ }^{66}$ L'artabe persiana viene considerata una misura (metron) per solidi (nel caso specifico l'argento), ma in Hom. Od. II 355 si parla di $\mu \varepsilon ́ \tau \rho \alpha$ di farina; il termine ha il senso di misura di capacità di un recipiente in cui si possono mettere acqua, vino, farina. L'impiego di queste misure fin dall'inizio è legato all'attività commerciale (cf. Grimaudo, Misurare, 149-50).

${ }^{67}$ Cf. Totelin, Pharmacopōlai, 77. Il talento attico pesava circa 26 kg., cf. Grimaudo, Misurare, 134.

${ }^{68}$ L'esempio è segnalato da Totelin, Pharmacopōlai, 77.

${ }^{69} \mathrm{Cf}$. l'ed. di Tagliapietra con ampio commento.

${ }^{70}$ Diminutivo di óvv $\xi$; si tratta del coperchio della parte superiore di una piccola conchiglia simile a quella della porpora (Dsc. II 8); questo opercolo bruciato produce un fumo aromatico. Un'altra 
quali molto diffuse. Ricordiamo le piante secondo l'ordine di esposizione dal $\mathrm{r}$. 1: $\mu \alpha \sigma \tau i ́ x \eta$ (mastice) $^{71}$ a cui si collega $\xi v \lambda o \mu \alpha \sigma \tau^{\prime} \chi \eta$ (legno di lentisco) ${ }^{72}, \sigma \tau \alpha \kappa \tau n$

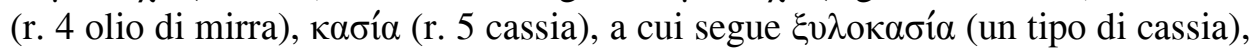

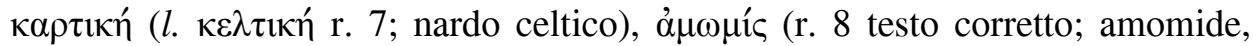

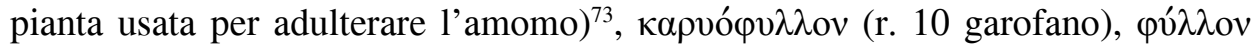

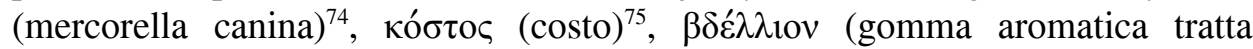
dall'albero del balsamo); ci sono però tre vegetali di non facile identificazione:

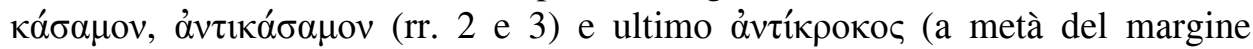
destro).

Per ciò che riguarda il $\kappa \alpha ́ \sigma \alpha \mu o v$, sulla base di un passo di Aetio che in una

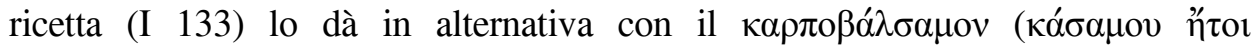

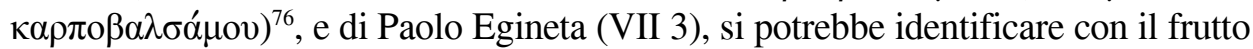
dell'albero del balsamo ${ }^{77}$, pianta aromatica e medicinale molto importante e, particolare significativo, proprio il frutto era soggetto ad adulterazione (Dsc. I 19,2 e 3) con un prodotto che veniva da Petra. Se accettiamo dunque per кó $\sigma \alpha \mu o v$ questo significato, $\alpha \dot{v} \tau \iota \kappa \alpha ́ \sigma \alpha \mu \nu$ (peraltro non attestato altrove) potrebbe voler indicare una sostanza sostitutiva ${ }^{78}$. Questa ipotesi si può avanzare sulla base di un parallelo con Dioscoride (V 75,13-16) dove si parla di sostanze ó $v \tau i ́ \sigma \pi o \delta \alpha$, che però hanno le stesse proprietà, da utilizzare quando manca la polvere, l'ossido di

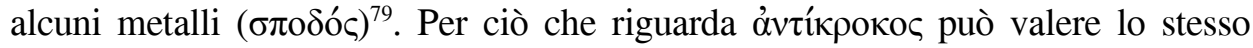
ragionamento, anche se lo zafferano è pianta molto nota e presente nei ricettari $(\text { vd. passim })^{80}$. I vegetali citati fanno parte delle piante profumate (aromata) e

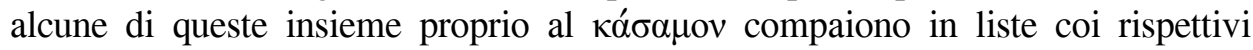

possibilità è che si tratti di un minerale, una varietà di onice, cf. Thphr. Lap. 31 .

${ }^{71}$ Dsc. I 70,3, lo identifica con la resina del lentisco ( $\left.\sigma \chi \hat{i} v o \varsigma\right)$.

${ }^{72} \mathrm{Nel}$ margine destro in alto; cf. per il significato il paragone con Dsc. I 19,3 dove $\xi \cup \lambda o \beta \alpha ́ \lambda \sigma \alpha \mu o v$ è il legno dell'albero del balsamo.

${ }^{73}$ Secondo Dsc. I 15,2.

${ }^{74}$ Dsc. III 125 indica con questo nome la mercorella canina (Mercurialis perennis L.). Secondo Ps.Gal. Succ. XIX 746,7 K. poteva sostituire sia lo spigonardo sia l'iris illirica.

${ }^{75}$ Saussurea lappa, se ne usa la radice per incensi e profumi; citata ad es. da Thphr. H.P. IX 7,3 e Dsc. I 16.

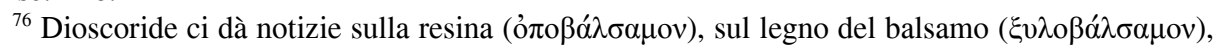
sul frutto e sull'origine della pianta che cresce solo in Giudea (I 19); Teofrasto ne parla diffusamente (H.P. IX 6,1-4) e anche Galeno menziona albero, resina e frutto, ad es. in Meth.med. X 466 K. o Comp.med. loc. XII 554 K.

${ }^{77}$ Cioè Commiphora opobalsamum L.; secondo André, Plants, 52 deriverebbe da una contrazione

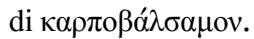

${ }^{78} \mathrm{O}$ magari adulterata, visto che si tratterebbe del frutto.

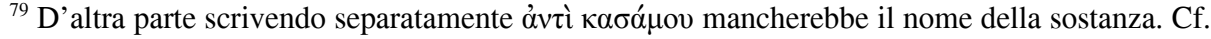
Tagliapietra, p.156.

${ }^{80} \mathrm{La}$ Tagliapietra segnala il riferimento ad una pianta acquatica con questo nome, peraltro non identificata, in Hippiatr.Paris. 712. 
prezzi dati dai venditori di profumi ( $\mu$ $\rho \circ \hat{\omega} \lambda \alpha$ ) in P.Oxy. LIV 3731 (310/1 d.C.), 3733 (312 d.C.), 3765,32-40 (ca. 327 d.C.), 3766v, 77-113 (327 d.C.) ${ }^{81}$.

Gli elementi di tipo minerale sono tutti usati in medicina o come pigmenti

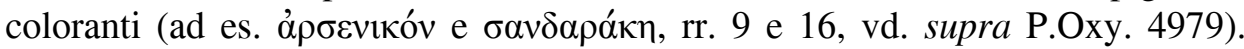
Invece un termine che non figura in opere di farmacologia o ricette su papiro è $\pi \alpha ́ \tau \eta \mu \alpha$ (r. 17), che indica qualcosa di "calpestato", cioè spezie pestate, ridotte in polvere ${ }^{82}$. Compare però in papiri documentari in una lista di prezzi (in P.Oxy. LIV 3733 del 312 d.C. e 3766 del 329 d.C.); in P.Oxy. VIII 1142, del III d.C., è

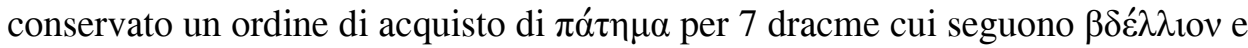
ővv $\xi$, presenti anche nel testo in esame.

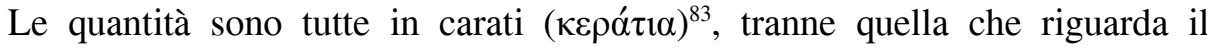
кó $\sigma \alpha \mu \mathrm{ov}$, dove si usa nomisma che equivaleva a 24 carati; si registra però metà

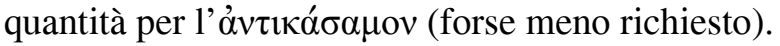

In conclusione, minerali a parte, si tratta di un elenco classico di piante profumate oggetto di fiorente commercio, in quanto in gran parte di origine orientale, come del resto si può constatare già nell'esposizione di Teofrasto (H.P. IX 7,3), che contiene piante tutte di provenienza orientale tranne l'iris ${ }^{84}$. L'argomento generale riguarda o un registro, come abbiamo visto nel caso di P.Oxy. 2567, oppure una lista di prodotti in vendita da parte di un $\varphi \alpha \rho \mu \alpha \kappa о \pi \omega ́ \lambda \eta \varsigma$

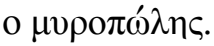

Su questa stessa linea si pone P.Oxy. 5253 del VI d.C. ${ }^{85}$; si tratta di un elenco molto breve, 6 righe con una lista di elementi tutti vegetali tranne l'ultimo: spigonardo, costo, cardamomo (si tratta di un'integrazione), garofano, zafferano e appunto "muschio" (di origine animale) ${ }^{86}$; le quantità sono date in grammi e solo per il cardamomo in carati. Difficile dire se sia una ricetta o una semplice lista redatta da un medico o da un farmacista; possiamo notare che come in altri casi tutti gli elementi di solito sono usati per la produzione di profumi ${ }^{87}$, come per es. il croco (Dsc. I 54) o il nardo (Dsc. I 62), quest'ultimo presente anche in Teofrasto (Od. 28). Un ulteriore confronto con Aetio (XVI 144), una ricetta per la fabbricazione di incenso, mostra la presenza di tutti i componenti tranne il cardamomo.

\subsection{I profumi}

Se gli aromata venivano spesso usati in medicina, è naturale incontrare testi in

\footnotetext{
${ }^{81}$ Possiamo segnalare costo, mastice, amomo, cassia.

${ }^{82}$ Ipotesi di Tagliapietra, p. 155.

${ }^{83}$ Un carato equivale a gr 0,187 .

${ }^{84}$ Quelle presenti nel papiro anche con loro derivati sono sei: cassia, nardo, balsamo, costo, mirra, lentisco.

${ }^{85}$ Edito da D. Colomo.

${ }^{86}$ Il muschio è una sostanza aromatica estratta dalla ghiandola addominale del mosco siberiano (moschus moschiferus, una specie di cervo) usata per profumi ed unguenti.

${ }^{87}$ Cf. Colomo, p. 157.
} 
cui viene descritta la preparazione di oli profumati con istruzioni precise, come nel P.Oxy. 5242 (II d.C.) ${ }^{88}$. Sappiamo che gli unguenti profumati erano preparati dai $\mu \nu \rho \varepsilon \psi o$, letteralmente "coloro che cuociono i profumi", cioè li preparano aggiungendo materie grasse agli oli essenziali (unguentarii in latino). Dioscoride li nomina in quattro passi (I 20,1 e 109,4; III 4,3 e IV 23,2) parlando degli elementi che usano per rendere densi gli unguenti e anche Teofrasto ricorda la loro attività (H.P. IV 2,6; C.P. IV 14,11 e VI 19,3; frr. 4,8, 4,30, 4,58). In parallelo esistevano i venditori ( $\mu \nu \rho \circ \pi \hat{\omega} \lambda \mathrm{ol}$ ), citati da Teofrasto (frr. 4,40 e 4,45) e varie volte da Galeno nel De antidotis (ad es. XIV 24 o XIV 53 K.). In questo contesto vengono date istruzioni per la $\sigma \tau \hat{\psi} \psi 1 \varsigma$, l'addensamento, che era il primo passo per la preparazione degli oli profumati. La componente fondamentale nel papiro è l'olio di olive acerbe. La ricetta è introdotta dalla formula $\alpha^{\prime} \lambda \lambda \eta \sigma \tau \hat{\jmath} \psi 1 \varsigma$ che ci fa capire che prima era stato descritto un altro metodo, secondo un sistema espositivo diffuso ${ }^{89}$. Gli oli in preparazione vengono aromatizzati con tre piante fiorite: iris (rr. 1-12) ${ }^{90}$, rosa (rr. 13-22) e giglio (rr. 22 ss.) ${ }^{91}$ : sono tutti presenti in Dioscoride, anche se non trattati in maniera identica ${ }^{92}$ e inoltre il papiro seguiva probabilmente l'ordine alfabetico.

Un punto interessante sono le unità di misura impiegate per il peso, che sono molto particolari ( $\mu$ ńtıov e $\alpha ́ \sigma \alpha \rho o v)$ e non consentono paralleli convincenti. L'editore (p. 116) riporta l'ipotesi di W. B. Henry, che si tratti di un sistema di

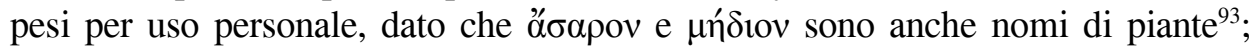
tuttavia almeno per $\mu$ ńtıov si può pensare che sia una forma errata per $\mu$ ó́tıv, misura egiziana di capacità, che però potrebbe adattarsi ad indicare la quantità della pianta profumata qui in questione, il calamo $^{94}$, misurata a volume, cioè secondo il contenitore che veniva riempito. Un possibile confronto con questa misurazione di quantità è con P.Oxy. 2567 (vd. supra) dove la possibilità è valida

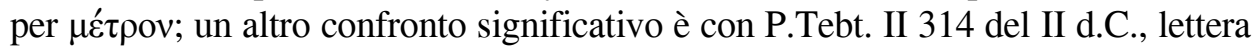
di Cherea, che chiede al fratello di mandargli degli abiti e anche tre $\mu$ ótı di

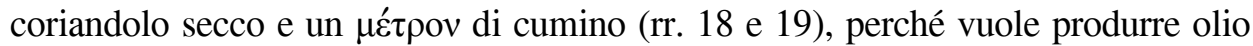

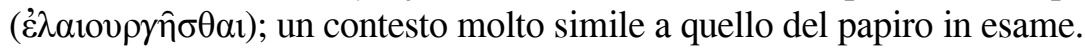

\section{Medicina magica}

Sei brevissime prescrizioni che si possono considerare di tipo magico sono

\footnotetext{
${ }^{88}$ Edizione di D. Leith. Nella letteratura medica Dioscoride (I 30-63) tratta accuratamente la preparazione di unguenti profumati.

${ }^{89} \mathrm{Si}$ veda l'esempio di P.Oxy. 1088 (vd. supra, n. 4).

${ }^{90} \mathrm{Il}$ nome della pianta è perduto, ma lo possiamo ricavare da Dsc. I 56, cf. Leith, p. 116.

${ }^{91}$ Rispettivamente in Dsc. I 43 e I 52.

${ }^{92}$ Quindi il testo non è quello di Dioscoride; si può pensare a una fonte comune, cf. Leith, p. 114.

${ }^{93}$ Dsc. I 10 (asaro) e IV 18 (campanula).

${ }^{94}$ Acorus calamus L., Dsc. I 18.
} 
riportate in P.Oxy. 5245, del II d.C. ${ }^{95}$, dove le foglie di alcune piante non sono utilizzate per comporre un rimedio, ma per essere portate addosso ( $\varphi$ ó 13, l. $\varphi$ ó $\rho \varepsilon)^{96}$. Nella prima ricetta infatti, si consiglia una foglia di piantaggine

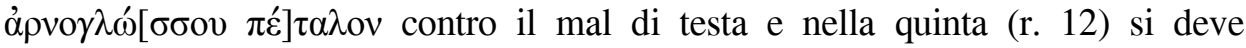
indossare contro la pesantezza di testa dovuta all'ubriachezza una specie di collana di foglie di dafne racemosa (o lauro di Alessandria) 'A $\lambda \varepsilon \xi \alpha \nu \delta \rho \varepsilon i ́ \alpha \varsigma \mid \chi \alpha \mu \alpha i \delta \alpha ́ \varphi] v n \varsigma$

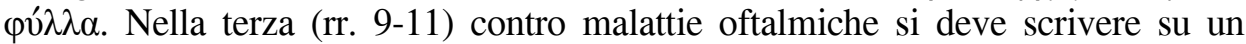
piccolo pezzo di papiro ( $\chi \alpha \rho \tau i ́ o v$ - la lacuna non ci consente di sapere che cosa) e indossare ${ }^{97}$. Questa abitudine di indossare a scopo protettivo qualcosa su cui è stata scritta una formula magica compare anche in alcuni testi editi nel vol. LXXXII dei papiri di Ossirinco, ad es. nel P.Oxy. 5303, del III d.C., che ci conserva resti di

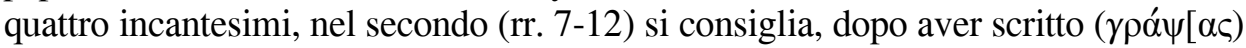

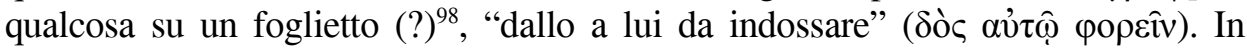
P.Oxy. 5304" indossando un amuleto (

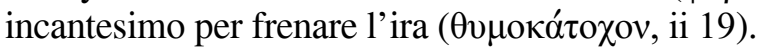

L'uso di scrivere su un pezzetto di papiro che diventa un amuleto grazie alle parole magiche è ampiamente testimoniato nei papiri egizi, come P.Oxy. XLII 3068 del III d.C..$^{100}$, dove lo scrivente chiede ad un suo interlocutore ignoto di mandare a Sarmate l'amuleto contro la tonsillite, scritto sulla lamina d'oro ( $\tau$ ò

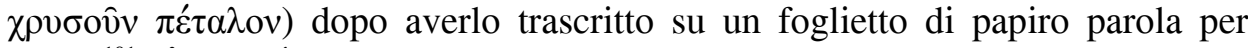
parola $^{101}$ (ஸ́ৎ $\left.\pi \varepsilon \rho l \varepsilon ́ \chi \varepsilon 1\right)$. Situazione analoga in PGM II 7, xvi 580-584:

Un filatterio per proteggere il corpo contro i demoni, i fantasmi, contro ogni malattia e sofferenza deve essere scritto su una lamina d'oro o d'argento o di

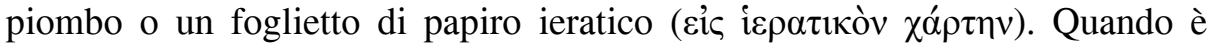

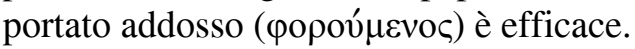

Da questi rapidi accenni si vede che oltre alla descrizione della composizione degli incantesimi, dove si impiegano sostanze vegetali, animali e minerali è interessante notare che viene specificato dettagliatamente il supporto scrittorio. ${ }^{102}$

\footnotetext{
${ }^{95}$ Edito da M. Hirt.

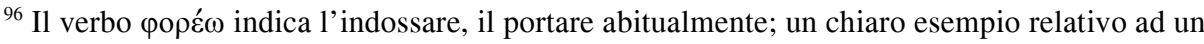
amuleto si trova nell'aneddoto di Galeno su un neonato di sei mesi che portava addosso

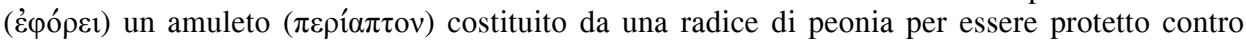
l'epilessia (Simpl.temp.fac. XI 859-860 K.). Cf. le osservazioni di Fausti, Farmacologia, 33.

${ }^{97}$ Un procedimento parallelo si incontra in Alessandro di Tralle (Febr. I 407 Puschmann): un amuleto contro le febbri quotidiane viene preparato raccogliendo una foglia di olivo prima del sorgere del sole, scrivendoci sopra delle lettere 'magiche' (' $\kappa \alpha$ ' ' $\rho \circ \iota^{\prime}$ ' $\alpha$ ') e appendendolo al collo

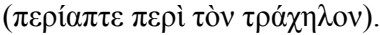

${ }^{98}$ Probabile integrazione dell'ed. Maltomini, p. 50.

${ }^{99}$ Ed. Maltomini.

${ }^{100}$ Edizione di Parsons, 1974. L'uso di amuleti era accettato sia negli ambienti egiziani sia greci, cf. Andorlini, Crossing, 171.

${ }^{101}$ Per assicurare l'effetto magico.

${ }^{102}$ Sull'argomento cf. le osservazioni di Minutoli, Exempla, 51-3.
} 


\section{Osservazioni generali}

Dal punto di vista linguistico i papiri di Ossirinco presi in esame ci testimoniano

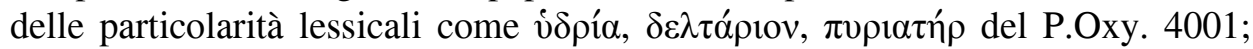
per ciò che riguarda le ricette, eccipienti e liquidi sono di uso comune come acqua, olio, aceto, miele, idromele e come la quasi totalità dei rimedi vegetali,

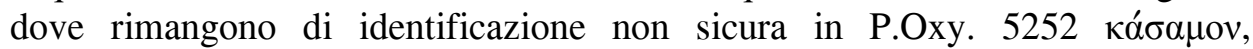

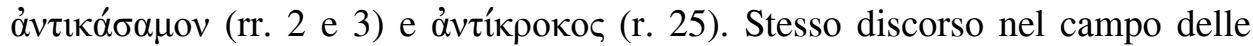
misure ponderali, dove abbiamo una notevole varietà di indicazioni: dracma, carato, nomisma, talenti, mine, panetti, libbre, once e kotylai e staio per olio e aceto. Nel P.Oxy. 2567 troviamo il non chiaro $\mu$ źt $\rho \alpha$ riferito ad un possibile modo di vendita della pianta a volume e nella lista del 5242 esempi di significato

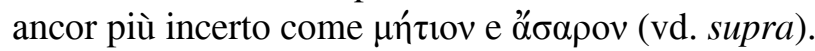

Il settore più interessante è costituito dai diminutivi, che compaiono con una

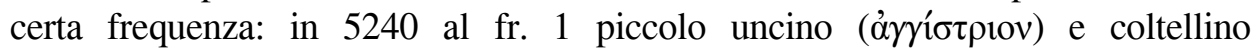
$(\sigma \mu \imath \lambda \alpha ́ \rho ı v)$, piccoli strumenti per chirurgia oculistica di precisione; un nuovo termine nel fr. 3,6: $\pi \varepsilon \rho$ tḯ́ $\delta$ เov, probabilmente un piccolo scalpello a lama curva (vd. supra), ma in complesso i diminutivi sono di uso abbastanza comune non soltanto per gli strumenti del medico, perché ci testimoniano i colloquialismi del linguaggio quotidiano. Possiamo ad esempio considerare in P.Oxy. 2567 il

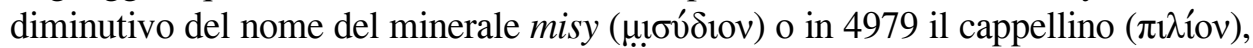
in 5245 foglietto di papiro ( $\chi \alpha \rho \tau i ́ o v)$ e in 5252 opercolo di conchiglia (ỏvó $\chi 10 v)^{103}$,

Si conferma ancora una volta la produttività della lingua d'uso dei papiri all'interno di testi medici o comunque tecnici.

\section{Bibliografia}

Andorlini, Borse = Isabella Andorlini, Le borse terapeutiche a vapore nella medicina antica, in: N. Reggiani (ed.), Medica-papyrologica, Specimina di ricerca presentati al convegno "Parlare la medicina" (Parma 5-7- sett. 2016), Parma 2016, 9-13.

Andorlini, Crossing = Isabella Andorlini, Crossing the Borders Between Egyptian and Greek Medical Practice, in: W. V. Harris (ed.), Popular Medicine in the Graeco-Roman World Antiquity, Leiden/Boston 2016, 161-72.

Andorlini, Strumenti = Isabella Andorlini, Gli strumenti perduti di Galeno, "La Torre di Babele" 8 (2012), 239-47.

Andorlini Marcone, Apporto = Isabella Andorlini Marcone, L'apporto dei papiri alla conoscenza della scienza medica antica, in W. Haase (ed.), Aufstieg und Niedergang der römischen Welt, II, 37.1, Berlin/New York 1993, 458-562.

André, Plants = Jacques André, Le noms des plantes dans la Rome antique, Paris 1985.

Bonati, Lessico = Isabella Bonati, Il lessico dei vasi e dei contenitori greci nei papiri. Specimina per un repertorio lessicale degli angionimi greci, Berlin/Boston 2016.

${ }^{103} \mathrm{Si}$ vedano per questi termini le osservazioni fatte nel commento ai testi. 
Braccini, Silfio = Tommaso Braccini, Il silfio nella tarda antichità: ancora su un passo del Gallus di Sulpicio Severo, "Quaderni Urbinati di Cultura Classica" 93 (2009), 161-75.

Fausti, Farmacologia = Daniela Fausti, La farmacologia antica: un ponte fra irrazionale $e$ razionale, in: V. Boudon-Millot / S. Buzzi (edd.), Guérison, religion et raison, De la médecine hippocratique aux neurosciences, Paris 2017, 17-39.

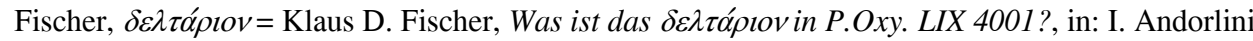
(ed.), 'Specimina' per il Corpus dei papiri greci di medicina, Atti dell'incontro di studio (Firenze, 28-29 Marzo 1996), Firenze 1997, 109-13.

Fischer Bovet, Letter $=$ Christelle Fischer Bovet, Letter Requesting Medical Tools, in: I. Andorlini (ed.), Greek Medical Papyri II, Firenze 2009, 157-65.

Ghiretti, Ambulatorio = Giulia Ghiretti, Ambulatorio e professione medica nell'Egitto grecoromano, in: N. Reggiani (ed.), Medica-papyrologica, Specimina di ricerca presentati al convegno "Parlare la medicina", (Parma 5-7- sett. 2016), Parma 2016, 79-100.

Ghiretti, Luoghi = Giulia Ghiretti, Luoghi e strumenti della professione medica antica. La testimonianza dei papiri greci d'Egitto, Parma 2010, URL: <http://dspace-unipr.cilea.it/handle/ $1889 / 1493>$.

Grimaudo, Misurare $=$ Sabrina Grimaudo, Misurare e pesare nella Grecia antica . Teorie storia ideologie, Palermo 1998.

Guash-Jané, Wine = Maria Rosa Guash-Jané, The Meaning of Wine in Egyptian Tombs: the three Amphorae from Tutankhamon's Burial Chamber, "Antiquity" 85 (2011), 851-8.

Kruse, Alaunmonopols $=$ Thomas Kruse, P. Heid. Inv. G 5166 und die Organisation des Alaunmonopols im kaiserzeitlichen Ägypten, in: J. Frösén / T. Purola / E. Salmenkivi (edd), Proceedings of the 24th International Congress of Papyrology, Helsinki, 1-7 August, 2004, I (Commentationes Humanarum Litterarum 122), Helsinki 2007, 523-47.

Lundon, POxy 1088 = John Lundon, POxy VIII 1088: problemi e proposte, in: I. Andorlini (ed.), Testi medici su papiro. Atti del seminario di studio (Firenze 2002), Firenze 2004, 119-30.

Manfredi, Referti = Manfredo Manfredi, Qualche osservazione sui referti medici nei papiri, in: I. Andorlini (ed.), Testi medici su papiro. Atti del seminario di studio (Firenze 2002), Firenze 2004, 153-70.

Minutoli, Exempla $=$ Diletta Minutoli, Exempla di vari supporti scrittori contenenti testi magici provenienti da Antinoupolis, in: M. De Haro Sanchez (ed), Écrire la magie dans l'antiquité, Actes du colloque international (Liège, 13-15 oct. 2011), Liège 2015, 51-67.

Parsons, Ossirinco = Peter Parsons, La scoperta di Ossirinco. La vita quotidiana in Egitto al tempo dei Romani, Roma 2014 [City of the Sharp-Nosed Fish, London 2007].

Reggiani, Report = Nicola Reggiani, Medical Report in: I. Andorlini (ed.), Medicalia Online, Parma 2016, URL: <http://www.papirologia.unipr.it/CPGM/medicalia/vocab/index.php?tema=171>.

Reggiani, Liquidi = Nicola Reggiani, Tra 'sapere' e 'saper fare': il problema della standardizzazione delle unità di misura dei liquidi nella testimonianza dei papiri greci d'Egitto, in: N. Reggiani (ed.), Medica-papyrologica, Specimina di ricerca presentati al convegno "Parlare la medicina", (Parma 5-7- sett. 2016), Parma 2016, 107-16.

Roselli, Silfio = Amneris Roselli, Breve storia del silfio, "Annali dell'Istituto Orientale di Napoli, Archeologia e Storia Antica" 8 (2001 [2004]), 11-20.

Totelin, Recipes $=$ Laurence M. V. Totelin, Hippocratic Recipes. Oral and Written Transmission of Pharmacological Knowledge in Fifth and Fourth-Century Greece, Leiden/Boston 2009.

Totelin, Pharmakopōlai = Laurence M. V. Totelin, Pharmakopōlai: A Re-Evaluation of the Sources in: W. V. Harris (ed.), Popular Medicine in Graeco-Roman Antiquity: Explorations, Leiden/Boston 2016, 65-85. 